and extended the lifespan of many to beyond 80 wks of age (figure $1 \mathrm{~B}$ and 1C). Safety assessments in C57BL6/J mice after 8 wks of treatment did not show any significant cardiac, behavioral, immunological and histopathological abnormalities. Alterations in serum chemistry analytes, including calcium and magnesium, but not markers of ketosis, were evident shortly after and persisted after 2DG exposure.

Conclusions The results highlight the potent and remarkable normalizing effect of intermittent 2DG therapy in the prevention and treatment lupus-like autoimmune disease with differing genetic and mechanistic etiologies and in its most precipitous forms. This mode of therapy did not incur untoward side effects. We thus propose that therapeutic inhibition of early steps in glycolysis by 2DG has broad potential for the treatment of lupus as well as related autoimmune disorders.

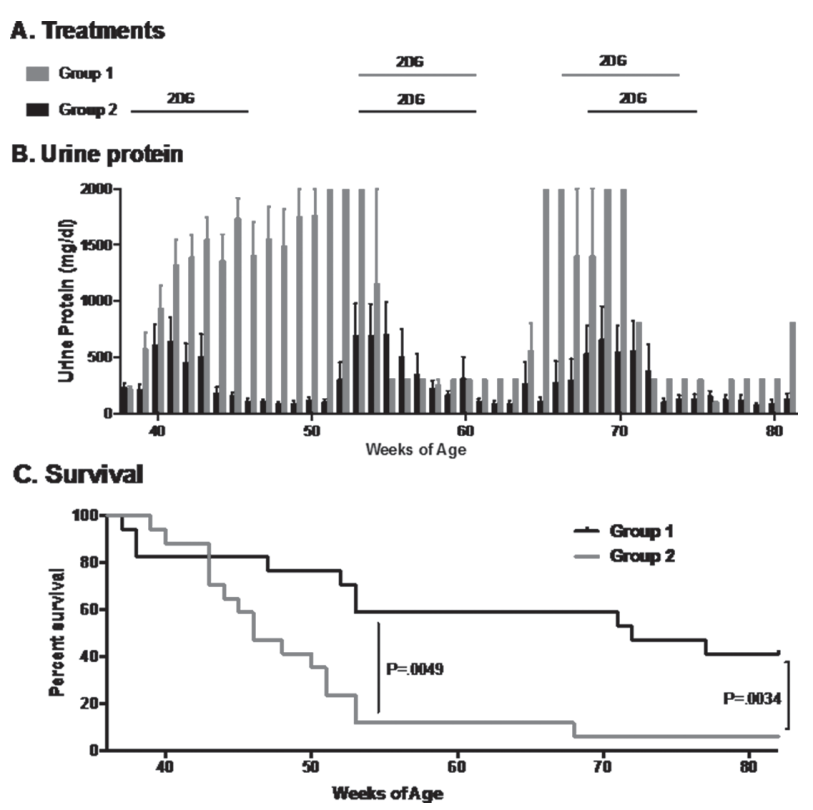

Abstract Al-03 Figure 1 Intermittent 2DG treatment reverses ongoing autoimmune disease of aged BWF1 mice. Twenty-nine BWF1 female mice aged to 37 wks were randomized into two cohorts and monitored to $>80$ wks of age. (A) one cohort was treated with 2DG for 8 wks starting at 37 wks. All surviving mice were then treated at 53 wk for 8 wks with 2DG and again treated at $67-70$ wks. (B) serial urine proteins. (C) overall survival.

\section{Al-04 IMMUNOTHERAPY FOR LUPUS IN A MOUSE MODEL TO DEFINE PATHOGENESIS AND THERAPEUTIC TARGETING}

${ }^{1}$ Hua Huang, ${ }^{2}$ Sophie Viaud, ${ }^{1}$ Argyrios N Theofilopoulos, ${ }^{2}$ Travis S Young, ${ }^{1}$ Dwight H Kono*. 'The Scripps Research Institute, La Jolla, CA, USA; ${ }^{2}$ California Institute for Biomedical Research, La Jolla, CA, USA

\subsection{6/lupus-2018-Ism.4}

Background Recent advances in immunotherapy using genetically modified chimeric antigen receptor (CAR) $\mathrm{T}$ cells have made it possible to selectively and completely eliminate cells expressing specific cell surface targets. Such a system could potentially be applied to lupus and other autoimmune diseases for identifying new targets in model systems and for therapy. To test the possible utility of such an approach, we therefore conducted a preliminary study to determine the efficacy of
anti-CD19 CAR T cell in eliminating B cells and disease development in a spontaneous mouse model of lupus.

Methods In these experiments, a modulatable CAR $\mathrm{T}$ cell system was used consisting of CAR $\mathrm{T}$ cells recognizing an antigenic site on a soluble anti-CD19 Fab 'switch.' Thus, activation and killing activity of CAR T cells was dependent on the presence of an independently injected anti-CD19 switch. To study CAR T cell efficacy, lupus-prone male BXSB mice at 3 months of age were initially conditioned with cyclophosphamide i.p., then 24 hour later given CAR T cells and either the anti-CD19 switch or PBS alone every other day (3 mice/group). Mice were followed for mortality, autoantibodies, proteinuria, and peripheral blood and spleen cells populations up to 9 weeks after CAR $\mathrm{T}$ cell transfer. Immunoglobulins and autoantibodies in sera were measured by ELISA. Flow cytometry was used to analyze immune cell populations and included a FITC-conjugated switch peptide to identify CARexpressing T cells. Proteinuria was determined by dipstick and kidney sections were PAS-stained and scored for glomerulonephritis on a $0-4$ scale.

Results An initial experiment documented that a single i.p. injection of $100 \mathrm{mg} / \mathrm{kg}$ of cyclophosphamide at 3 months of age did not reduce the development of lupus in BXSB male mice compared to PBS controls (4 and 3 mice/group). When mice treated with conditioning and CAR $\mathrm{T}$ cells were analyzed, the group given anti-CD19 switch but not PBS, had low levels of circulating $\mathrm{B}$ cells by one week after CART T cell transfer and for the duration of the experiment. Immunoglobulin and autoantibody levels were present in the PBS group, but undetectable in the anti-CD19 switch group at the end of the 9 week study. All PBS-, but none of the anti-CD19 switch-treated mice group developed severe glomerulonephritis (glomerulonephritis scores: $3.1 \pm 0.07$ vs $0.43 \pm 0.23, \mathrm{p}<0.001$ ). Conclusions In a small study, anti-CD19 CAR T cell treatment of lupus was highly effective in preventing the development of severe lupus glomerulonephritis. Strikingly, at 9 weeks after transfer, there was complete deficiency of circulating immunoglobulins suggesting that long-lived plasma cells either express sufficient levels of CD19 to be targeted by CAR T cells or less likely that the plasma cell population in lupus requires replenishment from newly generated B cells. These findings support the possibility of using the switchable CAR $\mathrm{T}$ cell approach to define the role of immune cell subsets in lupus and treatment of severe lupus.

Acknowledgements This work was supported by grants from the NHLBI, NIAMS, and NCI.

\section{AI-05 PLATELET RESPONSE TO IMMUNE COMPLEXES}

${ }^{1}$ Imene Melki, ${ }^{1}$ Nathalie Cloutier, 'Isabelle Allaeys, 'Genevieve Marcoux, ${ }^{1}$ Tania Levesque, ${ }^{1}$ Yann Becker, ${ }^{1}$ Nicolas Tessandier, ${ }^{2}$ Paul R Fortin, 'Eric Boilard*. ${ }^{1}$ Centre de Recherche du CHU de Québec - Université Laval, Faculté de Médecine de I'Université Laval, Département de microbiologie et immunologie, Québec, QC Canada; ${ }^{2}$ Centre de recherche du CHU de Québec - Université Laval, Faculté de médecine de I'Université Laval, Division de rhumatologie et Département de médicine, Québec, QC Canada

\subsection{6/lupus-2018-Ism.5}

Background There is a growing appreciation for the contribution of platelets to immunity; however, our knowledge mostly relies on platelet functions associated with vascular injury and the prevention of bleeding. Circulating immune complexes (ICs) contribute to both acute and chronic inflammation in a multitude of clinical conditions through their interaction with 